\title{
Development of a Modified Korean Version of the Epworth Sleepiness Scale Reflecting Korean Sociocultural Lifestyle
}

\author{
Seo-Yeon Jung ${ }^{1 *}$, Chul-Hyun $\mathrm{Cho}^{2,3 *}$, Min Kyu Rhee ${ }^{4}$, Leen $\mathrm{Kim}^{2,3}$, and Heon-Jeong Lee ${ }^{2,3} \bowtie$ \\ ${ }^{1}$ Department of Psychology, University of British Columbia, Vancouver, Canada \\ ${ }^{2}$ Department of Psychiatry, Korea University College of Medicine, Seoul, Republic of Korea \\ ${ }^{3}$ Korea University Chronobiology Institute, Seoul, Republic of Korea \\ ${ }^{4}$ Department of Psychology, Gyeongsang National University, Jinju, Republic of Korea
}

\begin{abstract}
Objective The Epworth Sleepiness Scale is a measure used for the diagnosis of sleep disorders including obstructive sleep apnea (OSA) syndrome, insomnia, and narcolepsy. Although a Korean version has been developed (the KESS), Korean lifestyle such as the floor culture and low driving rates has not been considered. We aim to develop and validate a modified KESS (mKESS) that reflects the Korean lifestyle.

Methods The sample consisted of 795 healthy participants and 323 OSA patients. The mKESS was developed by modifying several questions to concern the floor culture (questions 1,2,6, and 7) and low driving rates (question 8). Feasibility of the modification was tested by comparing the KESS and mKESS using paired samples t-test and by examining internal consistency reliability. Then, mKESS scores of the OSA patients and general participants were compared to test its validity.

Results Questions 1, 2, 7, and 8 were significantly different when comparing the performances of the general population on both scales. Especially, the mean scores on question 8 were significantly different in the non-driver group, but not in the driver group. Cronbach's alpha of the mKESS was relatively higher than that of the KESS. Total mKESS scores of the OSA patients were significantly higher than that of the general population.

Conclusion The mKESS is more universally applicable for the clinical evaluation of people that live in Korea. Results support that the mKESS can be administered to measure the average daytime sleep propensity of the Korean population as an alternative to the KESS.
\end{abstract}

Psychiatry Investig 2018;15(7):687-694

Key Words Epworth sleepiness scale, Modification, Sociocultural lifestyle, Korean.

\section{INTRODUCTION}

The Epworth Sleepiness Scale (ESS) ${ }^{1}$ is a self-rated scale developed in Australia, which numerically measures the respondent's subjective daytime sleepiness. It is also used for the diagnosis of sleep disorders [obstructive sleep apnea syndrome (OSAS), insomnia, and narcolepsy, etc.] where the total score from the questions helps identify daytime sleepiness of patients with sleep disorders. ${ }^{2}$ The questions consist of 8

\section{Received: March 8, 2018 Accepted: April 15, 2018}

$\triangle$ Correspondence: Heon-Jeong Lee, MD, PhD

Department of Psychiatry, Korea University Hospital, Korea University College of Medicine, 73 Inchon-ro, Seongbuk-gu, Seoul 02841, Republic of Korea Tel: +82-2-920-6721, Fax: +82-2-929-7679, E-mail: leehjeong@korea.ac.kr

*These authors contributed equally to this work.

(a) This is an Open Access article distributed under the terms of the Creative Commons Attribution Non-Commercial License (http://creativecommons.org/licenses/bync/4.0) which permits unrestricted non-commercial use, distribution, and reproduction in any medium, provided the original work is properly cited. different activities and situations that involve somnificity where the respondents are asked to rate their likeliness of falling asleep or dozing off in given situations. ${ }^{1}$ The total ESS score ranges from 0-24 where each question is rated on a 4-point scale (0-3). ${ }^{2}$ The ESS score of $0-10$ indicates normal daytime sleepiness and the score of 11-24 indicates a high level of daytime sleepiness where the respondent is recommended to seek medical attention. ${ }^{1,2}$ The ESS score of 16 or more is only found in patients with sleep disorders including narcolepsy, idiopathic hypersomnia, and OSAS. ${ }^{1}$

The strengths of the ESS are that it is relatively simple and free to administer compared to the other measures of sleepiness such as the Multiple Sleep Latency Test (MSLT) ${ }^{3}$ and Maintenance of Wakefulness Test (MWT). ${ }^{4}$ The MSLT and MWT are similar in nature in that both are performed in a laboratory setting, expensive, and time-consuming. ${ }^{3-5}$ In another aspect, the ESS is unique compared to another subjec- 
tive measure of sleepiness, the Stanford Sleepiness Scale (SSS). ${ }^{6}$ The ESS not only covers a wide range of sleep properties, but its score has significantly correlated with mean sleep latency (SL) determined by the MSLT., Although the ESS lacks objective strength, the scale provides a simple and standardized measurement of sleep propensity with high test-retest reliability and internal consistency.

The ESS has been translated and validated in many different languages for the evaluation of daytime sleepiness among patients with diverse cultural backgrounds. ${ }^{8-10}$ Many different language versions of the ESS including the Korean version have been developed by simply translating the original ESS. ${ }^{8,9,11-13}$ Although most translated versions have been standardized and validated, none of the studies noted that the original scale does not reflect a non-Western lifestyle. Therefore, the translated version of the ESS may not be appropriate to apply in many countries that do not practice a Western lifestyle. Because such modifications that only involve a simple translation method do not take into account cultural factors such as cultural and social differences, the ESS could either underestimate or overestimate the daytime sleepiness of non-western populations. A majority of Koreans practice the floor culture as people usually live at home with a floor heating system, called the "ONDOL."14 Koreans might find the ESS questions confusing given that some questions do not convey a concept of the floor culture. Moreover, the data collected by the Korean Statistical Information Service (KOSIS) showed that $93.4 \%$ of the population travel by public transportation, whereas only $4.1 \%$ of the total population travel by car. ${ }^{15}$ The data demonstrates that public transportation usage is high in Korea. Given that the majority of the population uses public transportation instead of private vehicles, the inclusion of an additional question is necessary to assess sleepiness in a non-driving population. According to the data collected by the Korea Transport Institute (KOTI), on average there were 1.9 licensed drivers and 1.5 personal vehicles per household. The mean number of drivers who drive more than once a month was 1.3 per household. ${ }^{16}$ The data illustrates that private car use (or driving prevalence) might not be high among licensed drivers. This further implies that people in Korea use alternative transportation methods more often than they use personal vehicles.

As sleep specialists, we have experienced that some of the questions from the ESS are insufficient to reflect the sociocultural background of Korea. Some modifications are necessary to improve the measurement accuracy of the ESS when administered to the Korean population. Most of the respondents were either confused or hesitant to answer certain questions that refer to situations involving sitting and driving. Ambiguity in interpreting these questions may arise because some Koreans tend to imagine themselves sitting on the floor when asked to think about a certain situation that involves sitting. For these reasons, we aimed to develop a Korean version of the ESS that concerns the Korean lifestyle, such as the practice of the floor culture and less self-driving.

Our research involved developing the modified Korean version of the ESS (mKESS) and testing its feasibility. The KESS $^{17}$ does not take into consideration the floor culture practiced by Japanese and Korean populations because it only reflects the Western lifestyle. Hence, when translated into Korean, some questions are not clear about whether respondents are to imagine themselves sitting on the floor or on a chair. Because sitting on the floor is different from sitting in a chair, in terms of resting position a person's chances of dozing off would be different depending on a person's posture. For this reason, we clarified the question by asking the respondents to imagine themselves sitting "on a chair or a sofa" in such situations. We also changed the last question of the KESS because it could underestimate the excessive daytime sleepiness of those who travel by public transportation or non-drivers.

\section{METHODS}

The questions from the original scale involve eight different situations that people encounter in everyday life. Questions 1 to 8 involve the following situations: "sitting and reading," "watching TV," "sitting, inactive in a public space," "as a passenger in a car for an hour without a break," "lying down to rest in the afternoon when circumstances permit," "sitting and talking to someone," "sitting quietly after a lunch without alcohol," and "in a car, while stopped for a few minutes in the traffic." These questions are listed in the order as presented in the scale. In consideration of the Korean lifestyle, we modified five questions that have references to sitting and driving. Questions 1,2,6, and 7 were specified as sitting on a chair or sofa in consideration of those who practice the floor culture. Question 8 was modified to concern both driving and nondriving populations. Question 8 covers a commuting situation involving public transportation that applies to both drivers and non-drivers. The scale was modified and translated into Korean by two sleep specialists and a bilingual Korean who majored in English literature and psychology. Forward and backward translation methods were used to ensure that linguistic and conceptual equivalence to the original scale was obtained. For questions 1, 2, 6, and 7, we specifically asked the respondents to imagine themselves sitting "on a chair or a sofa" and rate the likelihood of dozing off or falling asleep in certain circumstances. For question 8 , we asked the respondents to imagine themselves standing on public trans- 
portation and rate the chance of dozing off or falling asleep. The modified questions-questions 1, 2, 6, 7 and 8-were stated respectively as follows: "sitting on a chair and reading (e.g., a book, newspaper, magazine, or a document)," "sitting on a sofa (or a chair) and watching TV," "sitting on a chair and talking to someone," "sitting on a chair quietly after a lunch without alcohol," and "as a passenger in a vehicle (e.g., a bus or a train) holding a handle or leaning on a chair without alcohol" (Supplementary Material 1 and 2 in the onlineonly Data Supplement).

We also asked the respondents whether they drive or not as a means of categorizing them into the two groups of drivers and non-drivers.

\section{Participants}

The recruited participants were divided into three groups. First, a total of 800 general participants were recruited to test the reliability and validity of the mKESS. The general participants were selected proportionally with respect to age (ranging from 10-80 years old), gender, and region (urban and rural areas). From this sample, five participants were excluded as they failed to provide answers to all questions. By asking whether they are self-drivers, the participants were divided into two groups: drivers and non-drivers. Among the 795 participants, there were 421 drivers and 374 non-drivers. This procedure was intended to test the feasibility of question 8 on the mKESS, by comparing the mean score on the item to that of the KESS in the two different groups.

We then recruited 323 patients diagnosed with OSA (73 mild, 88 moderate, and 162 severe OSA) using the nocturnal polysomnography (PSG) from the Korea University Anam Hospital Sleep Center. The OSA patients were administered the mKESS and asked whether they are drivers or non-drivers. Lastly, 20 healthy participants were recruited separately for the assessment of test-retest reliability.

The participants were provided with written informed consent before the enrollment. The current study was approved by the Institutional Review Board of Korea University Anam Hospital (ED14241) and has followed the Declaration of Helsinki.

\section{Assessments}

Demographic data including age, gender, and body mass index was collected from all participants (Table 1). The KESS and mKESS were administered to the general population for the comparison of the original and the modified scales. The OSA patients were given the mKESS after testing the feasibility of the KESS and mKESS in the general population. The nocturnal PSG was performed on the patients for the diagnosis of OSA. The OSA patients were divided into 3 groups (mild, moderate, and severe) based on the severity of the OSA, which is determined by the PSG findings including the apnea-hypopnea index (AHI). PSG findings are shown in Table 2. Participants with an AHI score in the range of 5-14 were classified into the mild group, those with score of 15-29 were classified into the moderate group, and those with an AHI score of 30 or higher were classified into the severe group. In addition, 20 healthy participants were randomly drawn from the local community to evaluate test-retest reliability. The mKESS was administered twice at an interval of 3 weeks. The questionnaire and PSG were both performed and interpreted by the sleep medicine professionals and welltrained research staff.

Table 1. Demographic data of the general participants

\begin{tabular}{lccc}
\hline \multicolumn{1}{c}{ Participants } & Total $(\mathrm{N}=795)$ & Drivers $(\mathrm{N}=421)$ & Non-drivers $(\mathrm{N}=374)$ \\
\hline Age (year) & $44.42 \pm 19.73$ & $45.96 \pm 13.82$ & $42.69 \pm 24.67$ \\
Age distribution & 109 & 8 & 101 \\
$10 \mathrm{~s}$ & 118 & 50 & 68 \\
$20 \mathrm{~s}$ & 120 & 84 & 36 \\
$30 \mathrm{~s}$ & 119 & 94 & 25 \\
$40 \mathrm{~s}$ & 120 & 94 & 26 \\
$50 \mathrm{~s}$ & 109 & 77 & 32 \\
$60 \mathrm{~s}$ & 58 & 14 & 44 \\
$70 \mathrm{~s}$ & 39 & 0 & 39 \\
$80 \mathrm{~s}$ & 3 & 0 & 3 \\
$90 \mathrm{~s}$ & $22.48 \pm 3.18$ & $22.66 \pm 3.01$ & $22.27 \pm 3.35$ \\
BMI $\left(\mathrm{kg} / \mathrm{m}^{2}\right)$ & $50.2 / 49.8$ & $61 / 39$ & $38 / 62$ \\
Gender $(\mathrm{M} / \mathrm{F})(\%)$ & & \\
\hline
\end{tabular}

Values are presented in mean \pm SD. BMI: body mass index 


\section{Statistical analysis}

Statistical analysis of the study cohort was performed using SPSS 18.0 for Windows (SPSS Inc., Chicago, IL, USA). Psychometric methods such as paired/independent samples t-test and reliability analysis were used to evaluate the reliability and validity of the mKESS.

First, performance of the general participants on questions $1,2,6,7$, and 8 between the KESS and mKESS was analyzed using paired samples t-test. The corresponding questions from both scales were paired for the comparison of the mean scores. There were five pairs in total: questions 1, 2, 6, 7, and 8 of the KESS and mKESS. The reliability of both scales was examined with Cronbach's alpha. Item-total statistics were also analyzed for an accurate comparison between the KESS and mKESS.

We also conducted a comparative analysis of the items according to the driving status of the participants. Paired samples t-test was performed to compare the mean scores of the original and modified question 8 in the driver and non-driver groups. A key objective of this test was to determine which question was more reflective of the Korean lifestyle when applied to the groups of drivers and non-drivers. We intended to substantiate inclusion or exclusion of the questions for the mKESS based on the results.

Paired samples t-test was performed separately to assess test-retest-reliability of the mKESS with the scores obtained from the 20 healthy participants. Then, the mKESS scores of the OSA patients and the general participants were analyzed and compared to test the validity of the mKESS using oneway ANOVA and independent samples t-test. One-way ANOVA was performed for the comparison of the mKESS scores among the general participants and each of the OSA groups, and independent samples t-test was performed for the comparison of the mKESS scores between the general participants and OSA patients. All statistical analyses were twotailed, and the level of statistical significance was set at $\mathrm{p}<0.05$.

\section{RESULTS}

\section{Participants' characteristics}

Participants' demographics and polysomnographic findings are shown in Tables 1 and 2. Seven hundred ninety-five participants were recruited as the general population and the rest $(n=323)$ were patients with OSA. The mean age of the general population was $44.42 \pm 19.73$ (mean \pm SD), and the male to female percentage was $50.2 \%$ to $49.8 \%$. The mean age of the self-drivers was $45.92 \pm 13.82$, and the proportion of male participants was $61 \%$. The mean age of the non-drivers was $42.69 \pm 24.67$, and the male to female percentage was found to be $38 \%$ to $62 \%$.

Among the 323 OSA patients, 162 were diagnosed with severe OSA, 88 with moderate, and 73 with mild OSA (mean age $=48.71 \pm 16.0,68.7 \%$ male). The mean AHI of the OSA groups was $7.04 \pm 3.11,20.81 \pm 11.73$, and $49.53 \pm 16.94$ for the mild, moderate, and severe OSA groups, respectively.

\section{Comparison of the questions from the KESS and mKESS}

Results from the paired samples t-test between the questions of the KESS and mKESS in general participants are reported in Table 3. Among 5 pairs of questions $(1,2,6,7$, and 8) we found a significant difference for questions $1,2,7$, and $8(\mathrm{p}<0.05)$, but not for question $6(\mathrm{t}=-1.44 ; \mathrm{p}=0.151)$. In addition, there was a significant difference in the total scores

Table 2. Polysomnographic findings of obstructive sleep apnea (OSA) patients

\begin{tabular}{lccr}
\hline & \multicolumn{3}{c}{ OSA groups } \\
\cline { 2 - 4 } & Mild (N=73) & Moderate (N=88) & Severe (N=162) \\
\hline Apnea-hypopnea index & $7.04 \pm 3.11$ & $20.81 \pm 11.73$ & $49.53 \pm 16.94$ \\
Time in bed (min.) & $454.76 \pm 37.11$ & $446.56 \pm 79.72$ & $432.10 \pm 64.45$ \\
Total sleep time (min.) & $389.76 \pm 62.23$ & $361.69 \pm 81.65$ & $358.52 \pm 75.19$ \\
Wake time after sleep onset (min.) & $53.64 \pm 60.52$ & $72.97 \pm 66.68$ & $63.07 \pm 53.29$ \\
Sleep efficiency (\%) & $85.98 \pm 13.21$ & $81.59 \pm 13.32$ & $82.99 \pm 13.08$ \\
Sleep latency (min.) & $11.42 \pm 17.85$ & $11.86 \pm 13.62$ & $11.10 \pm 20.70$ \\
Stage R latency (min.) & $111.30 \pm 73.53$ & $113.54 \pm 65.89$ & $117.93 \pm 63.94$ \\
Sleep stages (\%) & & \\
Stage N1 & $21.50 \pm 12.25$ & $25.40 \pm 12.12$ & $41.67 \pm 17.09$ \\
Stage N2 & $49.56 \pm 11.48$ & $48.08 \pm 9.01$ & $37.75 \pm 13.02$ \\
Stage N3 & $9.48 \pm 7.77$ & $8.75 \pm 8.37$ & $5.22 \pm 7.52$ \\
Stage R & $19.47 \pm 6.00$ & $17.78 \pm 7.40$ & $15.39 \pm 6.43$ \\
\hline
\end{tabular}

Values are presented in mean \pm SD 
for the KESS and mKESS in the general participants $(t=3.04$; $\mathrm{p}=0.002$ ). The mean KESS total score was $6.62 \pm 3.61$, and the mean for the mKESS was $6.43 \pm 3.74$.

The paired samples t-test performed to compare the mean scores for question 8 between the KESS and mKESS among the drivers revealed no significant difference, $\mathrm{t}=-1.27, \mathrm{p}=0.206$ (Table 3); the mean scores were $0.50 \pm 0.69$ and $0.54 \pm 0.74$ respectively (mean $\pm S D)$. In contrast, the mean KESS score for question $8(0.35 \pm 0.60)$ was significantly different from the mean mKESS score for question $8(0.44 \pm 0.63)$ among the non-drivers, $\mathrm{t}=-2.75, \mathrm{p}=0.006$. In addition, there was a significant difference found for question 8 between the KESS and $\mathrm{mKESS}$ among the general population, $\mathrm{t}=-2.83, \mathrm{p}=0.005$; the mean scores were $0.43 \pm 0.65$ and $0.49 \pm 0.69$ respectively.

\section{Reliability statistics}

The results from the reliability analysis are presented in Table 4. The findings revealed that the KESS and mKESS were highly reliable. Internal consistency of the mKESS (Cronbach's $\alpha=0.805$ ) was slightly higher than that of the

Table 3. Paired samples statistics for the comparison of the questions between the KESS and mKESS in general participants

\begin{tabular}{|c|c|c|c|c|c|}
\hline & Mean & $\mathrm{SD}$ & $\mathrm{t}$ & df & $\mathrm{p}$ \\
\hline \multicolumn{6}{|c|}{ General participants $(\mathrm{N}=795)$} \\
\hline KESS Q1 & 0.95 & 0.70 & & & \\
\hline mKESS Q1 & 0.89 & 0.68 & 3.52 & 794 & $<0.001^{*}$ \\
\hline KESS Q2 & 0.87 & 0.70 & & & \\
\hline mKESS Q2 & 0.93 & 0.69 & -3.18 & 794 & $0.002 *$ \\
\hline KESS Q6 & 0.24 & 0.57 & & & \\
\hline mKESS Q6 & 0.26 & 0.60 & -1.44 & 794 & 0.151 \\
\hline KESS Q7 & 0.84 & 0.71 & & & \\
\hline mKESS Q7 & 0.79 & 0.70 & 2.90 & 794 & $0.004^{*}$ \\
\hline KESS Q8 & 0.43 & 0.65 & & & \\
\hline mKESS Q8 & 0.49 & 0.69 & -2.83 & 794 & $0.005^{*}$ \\
\hline KESS total score & 6.62 & 3.61 & & & \\
\hline mKESS total score & 6.43 & 3.74 & 3.04 & 794 & $0.002^{*}$ \\
\hline \multicolumn{6}{|c|}{ Drivers of the general participants $(\mathrm{N}=421)$} \\
\hline KESS Q8 & 0.50 & 0.69 & & & \\
\hline mKESS Q8 & 0.54 & 0.74 & -1.27 & 420 & 0.206 \\
\hline \multicolumn{6}{|c|}{ Non-drivers of the general participants $(\mathrm{N}=374)$} \\
\hline KESS Q8 & 0.35 & 0.60 & & & \\
\hline mKESS Q8 & 0.44 & 0.63 & -2.75 & 373 & $0.006^{*}$ \\
\hline
\end{tabular}

${ }^{*} \mathrm{p}<0.05$. KESS: Korean version of the Epworth Sleepiness Scale, mKESS: modified Korean version of the Epworth Sleepiness Scale

Table 4. Reliability analysis of the KESS and mKESS

\begin{tabular}{|c|c|c|c|c|c|c|}
\hline \multirow{2}{*}{$\begin{array}{c}\text { Item } \\
\text { number }\end{array}$} & \multicolumn{3}{|c|}{ KESS $(\mathrm{N}=795)$} & \multicolumn{3}{|c|}{ mKESS $(\mathrm{N}=795)$} \\
\hline & Mean \pm SD & $\begin{array}{l}\text { Corrected item } \\
\text { to total correlation }\end{array}$ & $\begin{array}{c}\text { Cronbach's alpha } \\
\text { if item deleted }\end{array}$ & Mean \pm SD & $\begin{array}{l}\text { Corrected item } \\
\text { to total correlation }\end{array}$ & $\begin{array}{c}\text { Cronbach's alpha } \\
\text { if item deleted }\end{array}$ \\
\hline 1 & $0.95 \pm 0.70$ & 0.534 & 0.782 & $0.89 \pm 0.68$ & 0.535 & 0.805 \\
\hline 2 & $0.87 \pm 0.70$ & 0.425 & 0.795 & $0.93 \pm 0.69$ & 0.492 & 0.810 \\
\hline 3 & $0.59 \pm 0.70$ & 0.568 & 0.777 & $0.54 \pm 0.68$ & 0.592 & 0.799 \\
\hline 4 & $1.23 \pm 0.88$ & 0.486 & 0.790 & $1.16 \pm 0.88$ & 0.523 & 0.809 \\
\hline 5 & $1.46 \pm 0.82$ & 0.403 & 0.800 & $1.36 \pm 0.82$ & 0.406 & 0.823 \\
\hline 6 & $0.24 \pm 0.57$ & 0.526 & 0.785 & $0.26 \pm 0.60$ & 0.542 & 0.806 \\
\hline 7 & $0.84 \pm 0.71$ & 0.507 & 0.785 & $0.79 \pm 0.70$ & 0.552 & 0.803 \\
\hline 8 & $0.43 \pm 0.65$ & 0.511 & 0.785 & $0.49 \pm 0.69$ & 0.608 & 0.797 \\
\hline Total & $6.62 \pm 3.61$ & & 0.776 & $6.43 \pm 3.74$ & & 0.805 \\
\hline
\end{tabular}

KESS: Korean version of the Epworth Sleepiness Scale, mKESS: modified Korean version of the Epworth Sleepiness Scale 
KESS (Cronbach's $\alpha=0.776$ ). Item-total statistics revealed that deletion of any of the questions including the modified ones would decrease the value of Cronbach's $\alpha$. Most of the questions from the mKESS had relatively higher item-total correlations when compared to that of the KESS.

The test-retest reliability of the mKESS was good as there was no significant difference between the total scores, $\mathrm{t}=-0.503$, $\mathrm{p}=0.621$. The mean score of the pre-test $(5.65 \pm 2.87)$ was not significantly different than that of the post-test (5.90 \pm 3.55$)$.

\section{Comparison of the mKESS scores between the general population and OSA patients}

The comparison analysis on the mKESS total score for the general participants and OSA patients yielded significant findings. The findings from the independent samples t-test are presented in Table 5. We found a significant difference in mKESS total scores between the general participants and OSA patients, $\mathrm{t}=-0.978, \mathrm{p}=0.029$. The total mKESS score for the OSA patients $(6.69 \pm 4.24)$ was significantly higher than that of the general participants $(6.43 \pm 3.74)$.

However, there was no significant difference between the total scores of the four groups as determined by a one-way ANOVA, $\mathrm{F}=0.547, \mathrm{p}=0.650$. Post hoc comparisons using Turkey's HSD indicated that the mean scores for the mild (6.86 \pm 4.18$)$, moderate (6.43 \pm 4.15$)$, and severe OSA groups $(6.76 \pm 4.33)$ were not significantly different from that of the general population $(6.43 \pm 3.74)$.

\section{DISCUSSION}

The purpose of the study was to develop a modified Korean version of the ESS and test its feasibility for the accurate assessment of daytime sleepiness in the Korean population. The study shares similar objectives with few other studies that have attempted to modify the ESS with regard to cultural differences. ${ }^{10,18}$ A rationale of these studies ${ }^{10,18}$ was that the ESS may not be suitable for patients with diverse living habits, which concurs with our assertion that a cross-cultural adaptation of the original scale was necessary.

Dr. Murray Johns has recently developed a new version of the ESS called the Epworth Sleepiness Scale for Children and Adolescents (ESS-CHAD) ${ }^{19}$ Minor modifications were made to the questions that have references to drinking and driving because such questions may not be appropriate for adolescents. ${ }^{19}$ Hence, questions 7 and 8 were replaced with "sitting quietly by yourself after lunch" and "sitting and eating a meal" correspondingly. Knowing that these adjustments were made to fit the specific population, modifications to the original ESS may be necessary when being applied to those from different social and cultural groups.

Onen et al. ${ }^{20}$ have also addressed that the scale requires modifications to improve the response rate among the elderly. The authors have examined the response pattern and found some questions require respondents to be socially and physically active enough to engage in certain activities such as driving. Question 8 was the most commonly unanswered question, which yielded a low response rate among older adults. ${ }^{20}$ Such findings further suggest that the scale may not be applicable to certain populations with different sociocultural patterns.

In the present study, the comparison of question 8 between the KESS and mKESS yielded different results based on whether or not an individual was a driver. There was no significant difference in the mean scores of question 8 between the KESS and mKESS when administered to the group of drivers. Given drivers have experience in using public transportation as well as in driving, they were capable of imagining themselves using public transportation and responding appropriately to the modified version of question 8 . Yet, the mean mKESS score for question 8 was significantly higher than the mean KESS score for question 8 in the non-driver group. Such a finding suggests that daytime sleepiness related to question 8 from the KESS may be underreported because the non-drivers had to take a drivers' perspective, which they are quite unfamiliar with.

The current study proposed modifications to the ESS that would reflect the Korean lifestyle concerning the floor culture and driving pattern. The reliability and validity of the modified scale were tested systematically to examine whether it measures daytime sleepiness in the Korean population more accurately than the original scale does. The results from this study showed that the modified scale could be more appropriate for the Korean population than the original scale. The content of the mKESS was linguistically validated to be similar to that of the KESS. Hence, a strong correlation between the original and modified scale was found from the item analysis. The mKESS was found to have high internal consistency and good test-retest reliability. These results from the reliability analysis and paired/independent samples t-test support our hypothesis that the suggested

Table 5. Comparison results of the mKESS total scores between the general participants and OSA patients

\begin{tabular}{lcccc}
\hline & General population $(\mathrm{N}=795)$ & OSA patients $(\mathrm{N}=323)$ & $\mathrm{t}$ & $\mathrm{p}$ value \\
\hline Total score of the mKESS $($ mean $\pm \mathrm{SD})$ & $6.43 \pm 3.74$ & $6.70 \pm 4.20$ & -0.978 & $0.029^{*}$ \\
\hline${ }^{*} \mathrm{p}<0.05$ & & & &
\end{tabular}


modifications enhance the reliability and validity of the original scale. The total mKESS score of the OSA patients was significantly higher than that of the general population, which suggests that the mKESS is capable of distinguishing OSA patients from the general population based on the total score. Hence, the finding verifies the construct validity of the mKESS. However, the mKESS was found to be relatively insufficient for distinguishing the severity of OSA, which can be explained with a low sensitivity of the original scale in the assessment of OSA severity. Previous studies have also reported insufficient sensitivity and specificity to distinguish the severity of OSA as one of the limitations of the ESS., ${ }^{5,17,21}$ Based on previous and present reports, it can be speculated that the mKESS may not be sensitive enough to distinguish severity of sleep-disordered breathing.

The current study has many strengths in terms of sample size, careful modification of the ESS questions to reflect Korean cultural practices, and the unique attempt to overcome cultural barriers in modifying the original scale. First, the study has a large sample size, which allowed us to perform extensive data collection from both the general population and OSA patients. It also allowed us to determine outliers and yield reliable results in testing the reliability and validity of the modified scale. Considering the sample size and control for demographic factors, it can be concluded that the general population is representative of the Korean population. Moreover, our study is unique in a sense that the scale has been modified to reflect cultural and social practices in Korea. Unlike other studies that attempted to modify the existing scale using a simple translation method, ${ }^{8,9,11-13}$ this research involved modifying the scale so that it would fit the Korean environment. The questions that required modification were carefully selected by examining the questions that yielded low response rates among the respondents. Based on other studies that aimed to modify the scale concerning cultural, economic, and social differences, ${ }^{11,17,18}$ we proposed specific modifications that convey the Korean lifestyle targeting the questions that yielded low response rates.

On the other hand, this study acknowledges several limitations of using a subjective scale. As a subjective scale that relies on self-report, the ESS requires the respondents to read, comprehend, and answer questions in an accurate manner. Daytime sleepiness can be either underestimated or overestimated depending on the subjective reports from the respondents. The validity of the scale can be affected by response bias as the ESS does not have a question that controls for bias in responding. ${ }^{21}$ Although the ESS lacking objectivity, including the scale with other objective scales such as the MSLT or MWT would improve the validity of the research. Administering the mKESS in hand with other objective scales would provide a more accurate diagnosis of sleep disorder and quantification of sleepiness. ${ }^{22}$ In addition, the recruitment methodology should be noted as another limitation of the study. The general population was not screened for other disorders when recruited. For this reason, we had to refer to this group of participants as the "general population" instead of "healthy participants." There is the possibility that the total mKESS score could have been affected by the presence of other disorders. Yet, the results from the comparison provide meaningful information about how OSA patients can be distinguished from the other groups of people in Korea by applying the mKESS.

Based on our findings, we conclude that the proposed modification improves the reliability and validity of the ESS. According to the results from the statistical analysis, questions $1,2,6,7$, and 8 of the mKESS were found to be similar to or more reliable than that of the KESS. Our findings support that the mKESS can be administered to measure the average sleep propensity of the Korean population as an alternative to the KESS. Given that the mKESS considers cultural specifics of the Korean environment, the use of the modified scale would be helpful to clinicians and researchers in Korea, in order to better examine daytime sleepiness of the Korean population. Further research is required to apply the mKESS developed from the current research to screen for other disorders and conditions that involve daytime sleepiness.

\section{Supplementary Materials}

The online-only Data Supplement is available with this article https://doi.org/10.30773/pi.2018.04.15.2.

\section{Acknowledgments}

We thank Dr. Murray Johns for his helpful comments and insightful discussions. This study was supported by the Korea Health 21 R\&D Project funded by the National Research Foundation of Korea (2017M3A9F1031220).

\section{REFERENCES}

1. Johns MW. A new method for measuring daytime sleepiness: the Epworth sleepiness scale. Sleep 1991;14:540-545.

2. Johns MW. Reliability and factor analysis of the Epworth Sleepiness Scale. Sleep 1992;15:376-381.

3. Littner MR, Kushida C, Wise M, G. Davila D, Morgenthaler T, Lee-Chiong T, et al. Practice parameters for clinical use of the multiple sleep latency test and the maintenance of wakefulness test. Sleep 2005;28: 113-121.

4. Mitler MM, Gujavarty KS, Browman CP. Maintenance of wakefulness test: a polysomnographic technique for evaluating treatment efficacy in patients with excessive somnolence. Electroencephalogr Clin Neurophysiol 1982;53:658-661.

5. Leng PH, Low SY, Hsu A, Chong SF. The clinical predictors of sleepiness correlated with the multiple sleep latency test in an Asian Singapore population. Sleep 2003;26:878-881.

6. Hoddes E, Zarcone V, Smythe H, Phillips R, Dement W. Quantification of sleepiness: a new approach. Psychophysiology 1973;10:431-436.

7. Johns MW. Daytime sleepiness, snoring, and obstructive sleep apnea: 
the Epworth Sleepiness Scale. Chest 1993;103:30-36.

8. Tsara V, Serasli E, Amfilochiou A, Constantinidis T, Christaki P. Greek version of the Epworth sleepiness scale. Sleep and Breathing 2004;8:91-95.

9. Banhiran W, Assanasen P, Nopmaneejumruslers C, Metheetrairut C. Epworth sleepiness scale in obstructive sleep disordered breathing: the reliability and validity of the Thai version. Sleep Breath 2011;15:571-577.

10. Zhang JN, Peng B, Zhao TT, Xiang M, Fu W, Peng Y. Modification of the Epworth sleepiness scale in central China. Qual Life Res 2011;20: 1721-1726.

11. Chen NH, Johns MW, Li HY, Chu CC, Liang SC, Shu YH, et al. Validation of a Chinese version of the Epworth sleepiness scale. Qual Life Res 2002;11:817-821.

12. Vignatelli L, Plazzi G, Barbato A, Ferini-Strambi L, Manni R, Pompei F, et al. Italian version of the Epworth sleepiness scale: external validity. Neurol Sci 2003;23:295-300.

13. Bloch KE, Schoch OD, Zhang JN, Russi EW. German version of the Epworth sleepiness scale. Respiration 1999;66:440-447.

14. Kim DK. The natural environment control system of Korean traditional architecture: Comparison with Korean contemporary architecture. Build Environ 2006;41:1905-1912.

15. (KOSIS) KSIS. Research on Commuting Patterns in Korea. Daejeon:
Korean Statistical Information Service (KOSIS); 2015.

16. (KOTI) TKTI. An investigation on usage patterns of vehicles in Korea. The Korea Transport Institute (KOTI) 2013;1-157.

17. Cho YW, Lee JH, Son HK, Lee SH, Shin C, Johns MW. The reliability and validity of the Korean version of the Epworth sleepiness scale. Sleep Breath 2011;15:377-384.

18. Takegami M, Suzukamo Y, Wakita T, Noguchi H, Chin K, Kadotani H, et al. Development of a Japanese version of the Epworth Sleepiness Scale (JESS) based on item response theory. Sleep Med 2009;10:556-565.

19. Janssen KC, Phillipson S, O'Connor J, Johns MW. Validation of the Epworth Sleepiness Scale for children and adolescents using Rasch analysis. Sleep Med 2017;33:30-35.

20. Onen F, Moreau T, Gooneratne NS, Petit C, Falissard B, Onen SH. Limits of the Epworth Sleepiness Scale in older adults. Sleep Breath 2013;17:343-350.

21. Olson L, Cole M, Ambrogetti A. Correlations among Epworth Sleepiness Scale scores, multiple sleep latency tests and psychological symptoms. J Sleep Res 1998;7:248-253.

22. Wise MS. Objective measures of sleepiness and wakefulness: application to the real world? J Clin Neurophysiol 2006;23:39-49. 